\title{
Polysèmes
}

Revue d'études intertextuelles et intermédiales

\section{Des fleurs qui poussent dans le silence : le langage de la couleur dans Alias Grace}

The Language of Color in Alias Grace: blood, cloth and the Letter

\section{Patricia Simonson}

\section{(2) OpenEdition \\ 1 Journals}

Édition électronique

URL : http://journals.openedition.org/polysemes/552

DOI : $10.4000 /$ polysemes. 552

ISSN : 2496-4212

Éditeur

SAIT

Référence électronique

Patricia Simonson, « Des fleurs qui poussent dans le silence : le langage de la couleur dans Alias Grace », Polysèmes [En ligne], 14 | 2015, mis en ligne le 18 novembre 2015, consulté le 10 octobre 2020.

URL : http://journals.openedition.org/polysemes/552; DOI : https://doi.org/10.4000/polysemes.552

Ce document a été généré automatiquement le 10 octobre 2020

Polysèmes 


\title{
Des fleurs qui poussent dans le silence : le langage de la couleur dans Alias Grace
}

The Language of Color in Alias Grace: blood, cloth and the Letter

\author{
Patricia Simonson
}

1 Le langage, et plus précisément la représentation verbale et non-verbale, est l'enjeu central du roman de Margaret Atwood. Cela tient au choix de son thème et personnage principal, la meurtrière présumée, Grace Marks. Ce personnage historique fut une cause célèbre dans le Canada colonial des années 1840 : il s'agit d'une jeune servante irlandaise, condamnée à la prison à perpétuité pour complicité dans le meurtre de son employeur, Thomas Kinnear, et de l'intendante et maitresse de celui-ci, Nancy Montgomery. Atwood souligne, dans son épilogue (Atwood 537-538), que Grace est une figure qui a réellement existé, et que l'auteur a reconstitué son histoire à partir de documents d'archives. On pourrait en déduire qu'il s'agit d'un roman historique; en réalité, c'est plutôt un roman métahistorique, qui remet en cause les prétentions de véracité $\mathrm{du}$ roman historique traditionnel ${ }^{1}$. Cette remise en cause est liée à la subversion des discours verbaux dominants - ceux de la religion, de la loi, de la médecine, de l'histoire - par le langage de la couleur, qui partout leur fait contrepoint, au point d'aboutir à un renversement des hiérarchies implicites : même si le visuel n'est jamais dissocié du verbal, qui en est presque toujours le support, cette dimension visuelle n'est pas simplement décorative. Elle constitue au contraire le principe d'organisation du récit à tous les niveaux, et propose ainsi le modèle d'une forme narrative alternative à la narration purement verbale. Nous allons voir que cela tient à l'omniprésence de la broderie et du patchwork, comme thème et comme structure : cet élément se constitue en système signifiant supra-verbal, destiné à contourner la dénotation linguistique, à la fois pour en suppléer les insuffisances et pour en contrecarrer les abus.

2 Le récit d'Atwood est un tissu, justement, de processus de représentation, qui maintiennent des rapports mutuels de dialogue, de conflit, et d'emboitement complexe. 
D'une part, comme le fait remarquer Tolan (255), le personnage de Grace est configuré par toute une série de discours écrits : biographies, récits journalistiques, extraits du règlement de la prison, etc. Des fragments de tous ces textes gravitent autour du récit principal sous forme d'épigraphes; par ailleurs, ils traversent la trame du roman, véhiculés par les discours des personnages. En même temps, un comité de notables locaux engage une sorte de contre-procès pour prouver l'innocence de Grace et obtenir sa libération. Dans ce contexte, une figure de psychanalyste en herbe (le docteur Jordan) essaiera d'éclaircir les détails du crime, dont la protagoniste nie le souvenir. Une partie considérable du roman est constituée par le discours de Grace en train de raconter sa vie à ce personnage. C'est ce discours-là qui nous concerne principalement ici $^{2}$.

3 Tous ces processus de représentation ont pour moteur quelque chose qui ne cessera de se dérober à toute tentative de nomination linguistique : il s'agit du rôle de Grace dans le meurtre de Nancy. D'après l'autre assassin, Grace aurait aidé à achever la victime ; quant à elle, elle prétend avoir perdu connaissance lors du crime et ne rien savoir de ce qui s'est passé (version que corrobore son monologue intérieur). Cet instant va donc rester inaccessible sur le plan cognitif et discursif, autant pour le lecteur que pour le personnage. Ce silence irréductible dans les souvenirs et dans le récit de Grace est le corrélat d'une négation plus générale: celle de son discours en tant que femme, servante, immigrée irlandaise dans une colonie britannique, et aussi en tant que prisonnière et à l'occasion patiente psychiatrique. Ainsi, Grace en tant que sujet social est entièrement niée par une série de discours dominants, qui l'enferment, au nom d'un moralisme bourgeois, religieux, et conservateur, dans une identité caricaturale.

Je vais tenter de montrer que les formes que prend la présence de la couleur dans le récit tendent justement à instaurer un contre-discours, qui substitue à la relation de signification discursive (transitive et référentielle) un acte de création nonréférentielle, quelque chose comme une irruption du sémiotique dans le symbolique, dans le sens où l'entend Kristeva dans La Révolution du langage poétique (Kristeva 1974). Le sémiotique et le symbolique sont les «deux modalités du procès de la signifiance, constitutif du sujet " (Kristeva 22), et correspondent, du moins en théorie, à deux stades successifs de la constitution du sujet. Le premier stade, pré-œdipien, serait celui des pulsions, antérieur à la signification et à la position du sujet (35); le symbolique, logiquement postérieur, est le stade de l'instauration du langage analytique, de la loi, du jugement (41). Néanmoins, ces deux stades en apparence hétérogènes coexistent et se complètent: "primitivement condition du symbolique, le sémiotique fonctionne dans les pratiques signifiantes comme le résultat de sa transgression» (67). Autrement dit, le pulsionnel, énergie privilégiée, non seulement de la psychose, mais de la création artistique, demeure en puissance au sein du langage discursif et référentiel, et peut donner lieu à une " explosion du sémiotique dans le symbolique " (68). À mon sens, le processus de création de Alias Grace, comme texte pluriel et éclaté (60), illustre particulièrement bien ce phénomène.

\section{Les fleurs rouges du silence}

Dans ses entretiens avec Jordan, Grace va être à la fois sujet et objet de son propre discours, récit « autobiographique » ou monologue intérieur. Ce discours très personnel devient un processus de configuration de soi de la part du personnage féminin, mais un 
processus hautement instable, et dont le résultat reste incertain ; c'est justement ce discours qui est régi par la présence de la couleur, sur le plan narratif, thématique et symbolique, d'un côté, et sur le plan métatextuel, de l'autre, dans la mesure où il s'agit de l'un des moteurs du récit ${ }^{3}$.

Commençons par voir comment fonctionne la couleur dans l'intrigue et le réseau symbolique du récit de Grace. Il faut dire avant tout que le regard du personnage est un regard d'artiste, qui perçoit avec une rare intensité les couleurs et les formes du monde physique qui l'entoure 4 . Sa perception des couleurs (réelle ou remémorée, c'est toujours, chez elle, un acte puissamment affectif) structure de manière vitale son expérience et le récit qu'elle en fait. Les moments clés de son existence sont constamment marqués par la couleur des objets qui y sont associés : les moufles bleu foncé, brodées de fleurs rouges, que son amie Mary Whitney lui offre, et la pochette à couture, fabriquée en tissu rouge, qu'elle-même donne à Mary en retour (Atwood 197); ou les pivoines blanches et les roses rouges qui ornent la véranda de Thomas Kinnear (241-242). Le spectacle de ces fleurs qui la frappe tellement à son arrivée chez son nouvel employeur ressemble à un écho inquiétant des fleurs blanches et du rouge de la pochette à couture que Grace, peu auparavant, a mis dans le cercueil de Mary (en cachette, nous dit-elle, parce que mélanger du rouge au blanc des fleurs funéraires ne convenait pas à la circonstance, Atwood 229) 5 .

7 À la fin du récit, lorsque Grace est enfin libérée, sa joie se traduit en termes sensoriels, notamment en termes de couleurs et de lumière :

I passed through the gates of the Penitentiary for the last time as the clock struck noon, and it went through my head like a thousand bells. Until that instant I couldn't quite trust my senses; while dressing for the journey I'd felt more numb than anything and the objects around me felt flat and lacking in colour, but now all sprang to life. The sun was shining and every stone of the wall seemed as clear as glass and lighted up like a lamp, it was like passing through the gates of Hell and into Paradise... (Atwood 518-519)

De même, lorsque Grace décrit sa vie avec son mari, tout le bonheur qu'elle retire de cette nouvelle existence semble se concentrer dans les couleurs: "The front of our house faces southwest and the sunlight is warm and golden [...]. On such days I think, This is like Heaven » (526). Les couleurs lumineuses (comme celle du chien, «the most beautiful shade of reddish-brown, like a polished chestnut ", 528-529) seraient donc l'équivalent du Paradis; même si le rouge, comme nous venons de le voir, joue à travers le roman un rôle instable.

En revanche, le noir renvoyant au costume du médecin qui pratiquera l'avortement de Mary $(32,203)$ devient bien sûr le signe de l'angoisse et du néant ${ }^{6}$. Nous voyons bien ici que la couleur n'est pas dénuée de sens, mais il ne s'agit pas d'une symbolisation linguistique : il s'agit de mettre en présence un monde d'émotions et de sensations qui exprime les expériences les plus fondamentales de la protagoniste, des expériences qui se dérobent au discours " analytico-référentiel» (Hutcheon 74 ; ma traduction) dans lequel les institutions sociales de son époque cherchent à l'enfermer.

Ce monde affectif et sensoriel va s'opposer, justement, aux discours religieux, juridiques et médicaux de la bonne société canadienne. Il fonctionnera, sur le mode onirique, comme ce qui permettra au personnage (aussi bien qu'au lecteur) de s'approcher d'un espace de silence interdit aux processus d'explication verbale qui tentent de le capter. Car le regard de Grace est également hanté par le souvenir de ces deux morts violentes qui ont eu lieu en sa présence et qui la concernaient de près : celle 
de Nancy et celle de Mary. La protagoniste affirme clairement (Atwood 206) que pour elle, cette dernière mort est un meurtre, commis à la fois par l'amant de Mary et par le médecin. Ces deux personnages féminins seront intimement associés dans les souvenirs de Grace et dans la trame du roman, puisque la mort de Mary apparaît comme la cause du meurtre de Nancy (et dans les deux cas, le rouge du sang versé est au centre de la scène); le vide dans les souvenirs de la protagoniste quant à la mort de Nancy donne lieu, de façon récurrente, à une vision hallucinatoire qui contribue dès le début du récit à suggérer l'association d'une certaine forme de création et de réception artistique, d'un côté, avec le sang et le corps féminin, de l'autre.

Pour comprendre ces dernières affirmations, il faut lire de plus près un fragment des toutes premières pages du roman. Grace est dans la cour de la prison, et nous raconte une sorte de «flashback » onirique lié au crime :

Out of the gravel there are peonies growing. They come up through the loose grey pebbles, their buds testing the air like snails' eyes, then swelling and opening, huge dark-red flowers all shiny and glossy like satin. Then they burst and fall to the ground. [...]

I watch the peonies out of the corners of my eyes. I know they shouldn't be here: it's April, and peonies don't bloom in April. There are three more now, right in front of me, growing out of the path itself. Furtively I reach out my hand to touch one. It has a dry feel, and I realize it's made of cloth.

Then up ahead I see Nancy, on her knees, with her hair fallen over and the blood running down into her eyes. Around her neck is a white cotton kerchief printed with blue flowers, love-in-a-mist, it's mine. She's lifting up her face, she's holding out her hands to me for mercy [...].

I am almost up to Nancy, to where she's kneeling. But I do not break step, I do not run, I keep on walking two by two; and then Nancy smiles, only the mouth, her eyes are hidden by the blood and hair, and then she scatters into patches of colour, a drift of red cloth petals across the stones. (Atwood 5-6)

Ces pivoines rouge sombre, qui fleurissent entre les graviers de la prison ou sur les murs blancs de la cellule, reviennent comme un leitmotiv à travers tout le roman. Un autre exemple très important de ce leitmotiv surgit dans le contexte du travail de remémoration au cours duquel la protagoniste a une vision où elle se rappelle sa chambre chez Thomas Kinnear (chambre qui se confond désormais avec sa cellule) :

[...] It's dark as a stone in this room, and hot as a roasting heart; if you stare into the darkness with your eyes open you are sure to see something after a time. I hope it will not be flowers. But this is the time they like to grow, the red flowers, the shining red peonies which are like satin, which are like splashes of paint. The soil for them is emptiness, it is empty space and silence. I whisper, Talk to me; because I would rather have talking than the slow gardening that takes place in silence, with the red satin petals dripping down the wall. (Atwood 344)

On voit ici une opposition apparente entre parole et image, qui privilégie la parole face à l'indicible, signifié par le rouge du sang et des pivoines qui fleurissent dans le silence de la cellule, et dans le silence intérieur de la mémoire perdue de la protagoniste. Néanmoins, cette opposition va s'estomper avec l'élaboration d'un langage composite, dans lequel le verbal s'imbrique dans le visuel à la manière des illuminations médiévales ou des poèmes gravés de Blake, d'une manière qui finira par inverser la hiérarchie initiale? 


\section{Patchwork et récit} manière plus générale, des tissus, tient au fait que Grace soit à la fois une meurtrière célèbre et une couturière de grand talent. Ces deux aspects du personnage s'avèrent interdépendants. Ils sont associés dès le début du récit: dans l'une des premières scènes du roman, où nous voyons Grace méditer sur son rôle « officiel » de meurtrière célèbre, elle déclare : « Sometimes at night I whisper it to myself. Murderess. Murderess. It rustles, like a taffeta skirt across the floor " (Atwood 25). On voit tout de suite ici, à petite échelle, l'appropriation de l'étiquette imposée par les discours dominants et sa transmutation en un signifiant autre, dépouillé de contenu sémantique précis et transposé à l'ordre du sensoriel. Précisons que le thème récurrent des tissus vêtements féminins, mouchoirs à fleurs, châles indiens couverts d'arbres et d'oiseaux est indissociable de celui du patchwork: en effet toute étoffe, surtout bariolée, peut être amenée à devenir matière première d'un nouveau tissu. D'une part, parce que les plus beaux morceaux des riches tissus apportés d'Europe étaient préservés, quand ils devenaient trop vieux, en étant découpés et cousus sur des courtepointes en patchwork; d'autre part, parce que les femmes, en fabriquant leurs édredons, y intégraient des fragments de vêtements anciens gardés pour leur valeur symbolique ou sentimentale ${ }^{9}$. Le patchwork est donc un palimpseste visuel des moments importants de la vie de sa ou de ses créatrice(s), et un système signifiant qui évoque sous forme chromatique un ensemble affectif (Chouard 73 ; Wilson 2003, 124-125).

En même temps, la dimension visuelle de cet art est toujours accompagnée d'une dimension verbale : les innombrables modèles et motifs d'édredons, créés et transmis par les femmes d'une génération à l'autre, reçoivent toujours des noms évocateurs (Cabane de bois, Étoile de Bethlehem, Vaisselle cassée...) qui renvoient à toute une tradition familiale, folklorique, littéraire ou biblique (Chouard 74 ; Wilson 2003, 125).

Dans le récit de Grace, la description des patchworks de la famille Parkinson (premiers employeurs de la jeune fille) est un moment essentiel du roman : ce passage confirme l'importance affective de ces objets pour la protagoniste, tout en soulignant leur lien implicite avec la mort, en particulier celle des femmes. Grace évoque, émue, la grande quantité et la beauté des courtepointes que ses employeurs possédaient; ce souvenir l'accompagne dans sa captivité: «Over the years in prison, when I have been by myself, as I am a good deal of the time, I have closed my eyes and turned my head towards the sun, and I have seen a red and an orange that were like the brightness of those quilts [...]» (Atwood 185). Puis, elle raconte la sensation qu'elle avait en voyant ces patchworks étendus, en train de sécher : il lui semblait que c'étaient les drapeaux d'une armée en campagne, placés par les femmes sur les lits comme un avertissement, le 
signe que le lit est le lieu du désir, mais aussi de la mort qui, pour les femmes du XIX siècle, est si souvent le prix de leur sexualité (Atwood 185-186) ${ }^{10}$.

Nous voyons que la broderie en général, et le patchwork en particulier, sont bien plus qu'un élément de couleur locale: leur fonction déborde à tous les niveaux la simple décoration, pour devenir un principe transversal d'organisation de tout le roman, autant narratif que symbolique. C'est particulièrement explicite si l'on examine la manière dont Atwood a disposé, et intitulé, les sections de son roman: le livre tout entier est physiquement présenté comme un patchwork. D'une part parce que le titre de chaque section évoque le nom d'un modèle de patchwork tout en désignant, souvent de manière oblique, les événements décrits dans la section en question (voir aussi Tolan 226). En même temps, chaque titre est illustré d'un carré qui représente un motif possible d'édredon. D'un côté, ces carrés peuvent se lire comme des motifs de bordure, qui accompagnent le texte principal; d'un autre, ils ressemblent aux carrés que l'on assemble pour fabriquer l'édredon tout entier, qui serait alors le roman lui-même : celui-ci peut donc se lire comme une étoffe composite en cours de fabrication (voir aussi Wilson 2003, 123).

Atwood superpose ainsi à la structure linéaire et discursive de son récit un principe d'organisation complètement autre, non-linéaire et non-discursif, qui ouvre le roman de tous les côtés et assure sa continuité avec toute la tradition du patchwork comme "texte" collectif $^{11}$. Quelque chose de semblable se produit grâce aux multiples références intertextuelles, par lesquelles le texte déborde partout ses propres limites (les épigraphes du roman incluent, au milieu des extraits de documents historiques, des fragments de poèmes et de romans) ${ }^{12}$. Une seule de ces références nous intéresse pour l'instant : celle qui intègre un classique de la littérature nord-américaine du XIXe siècle à la trame du roman d'Atwood. À un moment donné, deux personnages masculins commentent la tendance des dames à "broder», parlant, non de tissus, mais du discours féminin (Atwood 223). Dans la même page, comme par hasard (et sans rapport explicite avec le commentaire précédent), les mêmes personnages évoquent Nathaniel Hawthorne et son roman le plus connu, La Lettre écarlate. Ainsi, Atwood s'assure que le lecteur puisse faire le lien entre Grace et la plus célèbre brodeuse de la littérature américaine, Hester Prynne. Le roman de Hawthorne «brode» constamment sur la métaphore de la lettre pour faire du personnage un symbole de l'artiste ; quelque chose de semblable se produit pour Grace, avec une différence essentielle. En effet, la protagoniste d'Atwood reconnaît explicitement que son récit est une forme de broderie (elle arrange les faits pour faire plaisir à son psychanalyste...). Seulement, là où chez Hawthorne le personnage féminin et la broderie signifiaient l'artiste (et le discours) masculin, ici on pourrait dire que c'est le discours (féminin) qui signifie la broderie, c'est-à-dire le langage non-discursif ${ }^{13}$.

En effet, si le discours verbal de la protagoniste semble occuper la place centrale, il est constamment mis en doute en tant que discours référentiel. Ce n'est pas un discours de vérité, nous n'y trouverons jamais la réponse à la question sur ce qui s'est réellement passé. La "vérité » de son récit, c'est sa beauté formelle et son pouvoir d'évocation visuelle. Autrement dit, son discours devient une métaphore de la broderie, et non l'inverse, puisque la broderie est le véritable acte d'autocréation de Grace. C'est ce que confirment les toutes dernières lignes du roman. La protagoniste, libérée de sa prison après trente ans de réclusion, est en train de fabriquer enfin son propre quilt, un «Arbre du Paradis» : 
On my Tree of Paradise, I intend to put a border of snakes entwined; they will look like vines or just a cable pattern to others, as I will make the eyes very small, but they will be snakes to me; as without a snake or two, the main part of the story would be missing. [...] The Tree itself is of triangles, in two colours, dark for the leaves and a lighter colour for the fruits. They have many bright colours now, with the chemical dyes that have come in, and I think it will turn out very pretty.

But three of the triangles in my Tree will be different. One will be white, from the petticoat I still have that was Mary Whitney's; one will be faded yellowish, from the prison night-dress I begged as a keepsake when I left there. And the third will be a pale cotton, a pink and white floral, cut from the dress of Nancy's that she had on the first day I was at Mr. Kinnear's, and that I wore on the ferry to Lewiston, when I was running away.

I will embroider around each one of them with red feather-stitching, to blend them in as a part of the pattern.

And so we will all be together. (Atwood 534)

Il est à noter que si Grace est parvenue à se construire une identité au moyen de la broderie (et du récit), c'est une identité multiple, qui met en présence les trois principales figures féminines du roman, sur le mode, non de la fusion unitaire, mais du rapiècement. Nous retrouvons également le rouge, sous la forme du fil qui réunit les trois personnages; cela pourrait suggérer (mais le texte ne nous donnera aucune certitude à cet égard) que Grace s'est réconciliée avec sa propre sexualité, systématiquement réprimée tout au long de sa vie, et dont les deux autres jeunes femmes avaient récolté les fruits et fait les frais à sa place. En même temps, la réunion entre les trois personnages féminins, liées par des relations complexes de culpabilité réciproque - puisque Nancy apparaît comme coupable de son propre meurtre, dont Mary est l'auteur réel par l'intermédiaire physique de Grace (466-468) - instaure un espace de rencontre entre la vie et la mort, les victimes et les bourreaux. C'est d'autant plus le cas que Grace vient de nous annoncer (Atwood 533) qu'elle est, ou bien enceinte (comme l'étaient Mary et Nancy au moment de leur mort) ou bien atteinte d'une tumeur comme celle dont sa mère est morte. Autrement dit, elle se trouve elle-même à la frontière entre les deux mondes. Cette réitération de la présence du meurtre fondateur du roman au sein même d'un «happy end" ambigu rappelle le rôle de la mort dans le fonctionnement du langage, selon Kristeva, qu'il s'agisse de la dimension du sémiotique ou de celle du symbolique :

C'est au meurtre, à la mise à mort d'un homme, d'un esclave, d'un captif, d'un animal qu'incombe dans toutes les sociétés archaïques connues de représenter cette coupure instauratrice de l'ordre symbolique. [...] l'«art » assume et traverse le meurtre: il l'assume dans la mesure où la limite «mortelle» est placée, par la pratique artistique, comme limite interne au procès de la signifiance, une limite dont le franchissement précisément constitue l'« art »; en d'autres termes, la mort devient comme intériorisée par le sujet d'une telle pratique ; s'en faire le support lui est nécessaire pour fonctionner. (Kristeva 69)

Pourtant, la spécificité de l'artiste est de préserver la possibilité de mouvement entre le symbolique et le pulsionnel, et entre les différents langages artistiques où s'incarnent les pulsions : à mon sens, c'est cette ouverture, cette oscillation créatrice - inséparable, tout au long du récit, de ce que j'ai appelé le «langage de la couleur »- qui se trouve derrière l'incertitude dans laquelle le roman nous laisse quant au sort de Grace. « It is strange to know that you carry within yourself either a life or a death, but not to know which one " (Atwood 533) : lu sous l'angle du passage de Kristeva que je viens de citer, cette phrase décrit la dynamique de toute pratique signifiante et artistique. 


\section{BIBLIOGRAPHIE}

Arnheim, Rudolf. “A Plea for Visual Thinking”. Mitchell, W.J.T. (ed.). The Language of Images (1974). Chicago: U of Chicago P, 1980, 171-179.

Atwood, Margaret. Alias Grace. London: Virago, 1997.

Chouard, Géraldine. "L'Amérique comme patchwork ». Revue française d'études américaines 89 (juin 2001) : 70-85.

Dekker, George. The American Historical Romance. Cambrige : CUP, 1987.

Hutcheon, Linda. A Poetics of Postmodernism: History, Theory, Fiction. Routledge : New York, 1988.

Kristeva, Julia. La Révolution du langage poétique. Paris : Seuil, 1974.

Mitchell, W.J.T. (ed.). The Language of Images. Chicago: The U of Chicago P, 1980.

Mitchell, W.J.T. "Beyond Comparison: Picture, Text and Method". Picture Theory. Essays on Verbal and Visual Representation (1994). Chicago: The U of Chicago P, 1995, 83-107.

Nischik, Reingard M. "From Survivalwoman to Literary Icon: An Interview with Margaret Atwood". Engendering Genre: The Works of Margaret Atwood. Ottawa: U of Ottawa P, 2009, 253-276.

Peck, Amelia. American Quilts and Coverlets in the Metropolitan Museum of Art. New York: The Metropolitan Museum of Art/MQ Publications, 2007.

Simonson, Patricia. "Pictorial Cross-currents between Hawthorne and Atwood: Negotiating with the Dead". En cours d'évaluation pour publication dans la revue Perifrasis (Universidad de los Andes, Bogotá), 2015.

Teyssandier, Hubert. Les Formes de la création romanesque à l'époque de Walter Scott et de Jane Austen (1814-1820). Paris: Didier, « Études anglaises », 1977.

Tolan, Fiona. Margaret Atwood: Feminism and Fiction. New York: Rodopi B.V., 2007.

Wilson, Sharon Rose. "Sexual Politics in Atwood's Visual Art”. Margaret Atwood's Fairy-Tale Sexual Politics. Jackson: U of Mississippi P, 1993, 35-81.

Wilson, Sharon Rose. "Quilting as Narrative Art: Metafictional Construction in Alias Grace". Margaret Atwood's Textual Assassinations: Recent Poetry and Fiction. Sharon Rose Wilson (ed.).

Columbus: Ohio State UP, 2003, 121-134.

\section{NOTES}

1. Tel que l'incarne un auteur canonique comme Sir Walter Scott (voir Teyssandier 261-264; Dekker 29-31). Nous pourrions aussi parler de «métafiction historiographique », pour emprunter un terme à Linda Hutcheon (ix et passim ; ma traduction) : elle utilise ce concept pour caractériser un nouveau genre de roman historique postmoderne, qui questionne l'autorité d'un discours historique totalisant, prétendument «objectif» et indépendant de la position de l'énonciateur (93).

2. Dans ce sens, le roman d'Atwood illustre particulièrement bien les remarques de Hutcheon sur la manière dont la "métafiction historiographique» replace au centre du récit l'acte d'énonciation individuelle que le roman historique du XIX ${ }^{e}$ siècle reléguait en dehors du champ 
de la narration (voir Hutcheon, chapitre 5, "Contextualizing the Postmodern: Enunciation and the Revenge of "Parole'").

3. Les fils de ce tissu s'étendent bien au-delà des limites apparentes de l'histoire de Grace Marks, et nous relient à une multitude d'autres textes et auteurs : Scott, Hawthorne, Byron, Tennyson, Browning, Dickens, la Bible, les Mille et une Nuits, la mythologie grecque... Je reviendrai brièvement ci-dessous sur le lien entre Grace Marks et Hester Prynne ; mais j'ai consacré une étude beaucoup plus détaillée à la relation entre Alias Grace et The Scarlet Letter dans un article (voir Simonson 2015).

4. Plusieurs critiques ont déjà fait remarquer que la fiction d'Atwood puise une part importante de son inspiration dans l'art visuel, qu'elle pratique elle-même sous plusieurs formes aquarelles, caricatures, bandes dessinées, couvertures de certains de ses livres - depuis le début de sa carrière (voir par exemple Wilson 1993 ; Nischik 2009).

5. Mary, séduite et enceinte de son employeur, meurt vidée de son sang, à la suite d'un avortement clandestin. Dans ce sens, le rouge de la pochette convenait peut-être trop bien à la circonstance en question.

6. Ces associations de couleurs ne sont certes ni subtiles ni originales, mais ce sont les choix de l'auteur elle-même. Elles pourraient bien relever du goût d'Atwood pour le monde " pop » et sans nuances du comic, et seraient cohérentes avec le rejet qu'incarne Grace face à la culture bourgeoise, élitiste et conservatrice du Canada colonial.

7. Même si, bien entendu, le visuel n'est presque jamais réellement présent dans le roman d'Atwood, mais requiert la participation active de l'imagination du lecteur. L'article de Rudolf Arnheim, «A Plea for Visual Thinking " (Mitchell 1980, 171-179), apporte des éléments utiles pour cette réflexion sur l'importance de la perception visuelle évoquée dans Alias Grace, et la manière dont cette évocation remet en cause la priorité de la cognition discursive (bien que l'auteur persiste, de manière assez surprenante, à écarter sommairement le langage comme un espace de cognition: "words and sentences are only a set of references to facts that must be given and handled in some other medium », Arnheim, Mitchell 1980, 175). L'article de W.J.T. Mitchell, «Beyond Comparison: Picture, Text, and Method» (Mitchell 1994, 83-107) m'a également été très utile ici, avec sa distinction entre différents degrés de fusion entre le visuel et le verbal (une distinction qu'il conceptualise grâce aux concepts de « image/text », « image-text » et « imagetext»).

8. L'étude que fait Fiona Tolan de Alias Grace est pour l'essentiel riche et nuancée. Néanmoins, je ne partage pas sa vision pessimiste du patchwork : "In Alias Grace, the patchwork is an image of existential despair: a postmodern nightmare of the fragmented and meaningless self » (Tolan 242). Le postmodernisme d'Atwood, dans beaucoup de cas, me paraît bien plutôt ludique et jubilatoire.

9. Je remercie Géraldine Chouard pour les informations qu'elle m'a fournies oralement sur cet art, et pour les très belles images de patchworks qu'elle m'a procurées. Pour plus de renseignements sur l'art du patchwork dans l'Amérique du Nord au début du XIX ${ }^{\mathrm{e}}$ siècle, voir également Chouard; Peck.

10. Cette intuition préfigure, bien sûr, les circonstances de la mort de Mary Whitney.

11. Géraldine Chouard fait le lien entre ce mode d'organisation qui est celui du patchwork et la notion d'ensemble rhizomatique théorisée par Deleuze et Guattari : "Art de la juxtaposition soumis aux principes d'assemblage et d'hétérogenéité, le patchwork impose un régime de fragmentation, de contiguiité, qui dénonce toute unité organique, privilégie contact, contraste et différence » (Chouard 71).

12. Tolan (255) nous fait remarquer que les fragments de textes (citations, épigraphes) qui traversent le roman fonctionnent également comme les fragments de tissu qui composent le patchwork. Voilà qui pourrait encore contribuer à déstabiliser la distribution orthodoxe des 
fonctions entre les différents discours : du fait de leur fragmentation et dispersion à travers le roman, les citations deviennent l'équivalent de « textes » visuels.

13. Dans « Pictorial Cross-currents between Hawthorne and Atwood: Negotiating with the Dead » (Simonson 2015), je propose de lire Alias Grace (entre autres choses) comme une réécriture délibérée de The Scarlet Letter, qui critique et dépasse les limitations rencontrées par Hawthorne dans la commune entreprise d'autodéfinition de l'artiste américain.

\section{RÉSUMÉS}

L'article explore la fonction de l'omniprésence du visuel, et en particulier de la couleur, dans le roman de Margaret Atwood, Alias Grace. Cette dimension visuelle, qui passe par la référence à des arts populaires et féminins comme la broderie et l'art du contrepoint, constitue le principe d'organisation du récit à tous les niveaux (autant au niveau synchronique et métaphorique comme au niveau diachronique de la narration), à tel point qu'elle se constitue en une forme narrative hybride et expérimentale qui cherche à court-circuiter l'ordre discursif linéaire pour proposer un système signifiant supra-verbal: celui-ci servirait à la fois à suppléer les insuffisances de la dénotation linguistique et à en contrecarrer les abus.

The article explores the function played by the omnipresence of the visual, and especially of color, in Margaret Atwood's Alias Grace. This visual dimension, which involves references to popular and feminine arts like embroidery and patchwork, organizes the novel at all levels (at the synchronic and metaphorical level as well as the diachronic, narrative one), to the point that it produces a hybrid, experimental narrative form which seeks to by-pass linear discourse in order to propose a supra-verbal signifying system: this system would both fill the gaps in linguistic denotation and correct its abuses.

\section{INDEX}

Mots-clés : broderie, discours, texte/image, intermédialité, intertextualité, narration, patchwork, littérature comparée

oeuvrecitee Alias Grace

Keywords : embroidery, discourse, word/image, intermediality, intertextuality, narration, patchwork, comparative literature

\section{AUTEURS}

\section{PATRICIA SIMONSON}

Patricia Simonson (psimonson@unal.edu.co) possède une Maîtrise en Traduction et Stylistique Comparée, et un Doctorat en Littérature Américaine, de l'Université de Paris III-Sorbonne Nouvelle. Elle est professeur associée au Département de Littérature de l'Université Nationale de Colombie (Bogotá) depuis 2000. Ses principaux champs de recherche et d'enseignement sont la littérature nord-américaine et anglaise $\left(16^{\mathrm{e}}-19^{\mathrm{e}}\right.$ siècles, principalement) et la théorie littéraire 
(en particulier la sémiotique et la littérature comparée, avec un intérêt particulier pour les relations entre les arts, et entre la littérature et les autres discours sociaux). Elle a publié des articles dans ces domaines dans des revues françaises et colombiennes, ainsi que des traductions entre l'anglais, le français et l'espagnol. Elle a édité un livre d'essais en littérature comparée (Variaciones : seis ensayos de literatura comparada, Universidad Nacional de Colombia, Facultad de Ciencias Humanas, Bogotá, 2011), auquel elle a également contribué l'introduction et un article ("De ladrones y de monstruos », Simonson 2011, 181-234) sur la dissémination intertextuelle de l'esthétique de Schiller et de la narration de Sade dans Frankenstein, de Mary Shelley. Elle édite en ce moment un autre livre d'articles de littérature comparée, Babel, antes y después, basés sur le congrès de littérature comparée « Después de Babel, la literatura como discurso políglota » organisé à l'Université Nationale de Colombie en mars 2013, livre auquel elle a contribué l'article «Sentido y sentidos : arte, lenguaje y experiencia sensorial », et pour lequel elle prépare actuellement l'introduction. Elle est actuellement (juin 2012-juin 2014) directrice de son Département et directrice du groupe de recherche Contrapuntos : Investigaciones en literatura comparada. 